PROCEEDINGS OF THE

AMERICAN MATHEMATICAL SOCIETY

Volume 133, Number 12, Pages 3643-3646

S 0002-9939(05)08064-0

Article electronically published on June 2, 2005

\title{
A NONCOMMUTATIVE SZEGÖ THEOREM FOR SUBDIAGONAL SUBALGEBRAS OF VON NEUMANN ALGEBRAS
}

\author{
L. E. LABUSCHAGNE
}

(Communicated by David R. Larson)

\begin{abstract}
For almost forty years now the most frustrating open problem regarding the theory of finite maximal subdiagonal algebras has been the question regarding the universal validity of a non-commutative Szegö theorem and Jensen inequality (Arveson, 1967). These two properties are known to be equivalent. Despite extensive efforts by many authors, their validity has to date only been established in some very special cases. In the present note we solve the general problem in the affirmative by proving the universal validity of Szegö's theorem for finite maximal subdiagonal algebras.
\end{abstract}

We assume $\mathcal{M} \subset B(\mathfrak{h})$ (h a Hilbert space) to be a von Neumann algebra equipped with a finite normalised faithful normal trace $\tau$. At an intuitive level this construct of course fulfills the role of a non-commutative $L^{\infty}(\mathbb{T})$. For any subset $\mathcal{S}$ of $\mathcal{M}$ we will write $\mathcal{S}^{*}$ for the set $\left\{a \in \mathcal{M}: a^{*} \in \mathcal{S}\right\}$. Now given a von Neumann algebra $\mathcal{M}$ and a von Neumann subalgebra $\mathcal{N}$, an expectation from $\mathcal{M}$ onto $\mathcal{N}$ is defined to be a positive identity preserving linear operator $\Phi: \mathcal{M} \rightarrow \mathcal{N}$ which satisfies the property $\Phi(x y)=x \Phi(y)$ for all $x \in \mathcal{N}$ and $y \in \mathcal{M}$. A comprehensive survey of the fundamentals of the theory of expectations may be found in A, Section 6 . Within the above context the non-commutative analogue of $H^{\infty}(\mathbb{T})$ is given by the following:

Definition. Let $\mathcal{A}$ be a weak* closed unital subalgebra of $\mathcal{M}$, and let $\Phi$ be a faithful normal expectation from $\mathcal{M}$ onto the diagonal von Neumann algebra $\mathcal{D}=\mathcal{A} \cap \mathcal{A}^{*}$. We deem $\mathcal{A}$ to be a finite maximal subdiagonal subalgebra of $\mathcal{M}$ with respect to $\Phi$ if:

i) $\mathcal{A}+\mathcal{A}^{*}$ is weak $k^{*}$ dense in $\mathcal{M}$;

ii) $\Phi(x y)=\Phi(x) \Phi(y)$ for all $x, y \in \mathcal{A}$;

iii) $\tau \circ \Phi=\tau$.

Observe that in the present context a subalgebra $\mathcal{A}$ of the type defined above is automatically maximal among those subalgebras satisfying i) and ii) [E]. Given

Received by the editors August 18, 2004.

2000 Mathematics Subject Classification. Primary 46L52; Secondary 46E25, 46J15.

Key words and phrases. Subdiagonal algebra, non-commutative, Szegö's theorem, Jensen's inequality.

Part of this research was conducted with the support of a grant under the Poland - South Africa cooperation agreement.

(C)2005 American Mathematical Society Reverts to public domain 28 years from publication 
a finite maximal subdiagonal subalgebra of $\mathcal{M}$, we will by analogy with the classical setting write $H^{\infty}(\mathcal{M})=\mathcal{A}$ where convenient. By $H_{0}^{\infty}(\mathcal{M})$ we will denote $H_{0}^{\infty}(\mathcal{M})=\left\{a \in H^{\infty}(\mathcal{M}) \mid \Phi(a)=0\right\}$.

For algebras $\mathcal{M}$ of the type under consideration here, we may define the determinant of an element of $\mathcal{M}$ by

$$
\Delta(a)=\lim _{t \searrow 0} \tau\left(|a|^{t}\right)^{1 / t}
$$

if $a \in \mathcal{M}$ is invertible [A 4.3.6], and by

$$
\Delta(a)=\inf _{\epsilon>0} \Delta(|a|+\epsilon \mathbb{1})
$$

otherwise. In the case of an invertible element $a \in \mathcal{M}$, the fact that $\tau(\mathbb{1})=1$, combined with Hölder's inequality for non-commutative $L^{p}$-spaces, of course ensures that the $\tau\left(|a|^{t}\right)^{1 / t}$ 's decrease to $\Delta(a)$ as $t \searrow 0$. For a detailed description of the basic properties of this determinant we refer the interested reader to [A, §4.3].

Although the following statements regarding this determinant are not known to be universally valid for finite maximal subdiagonal subalgebras $\mathcal{A}=H^{\infty}(\mathcal{M})$ of $\mathcal{M}$, they are known to be equivalent for such algebras [A, 4.4.3]:

- Jensen's inequality: $\Delta(a) \geq \Delta(\Phi(a))$ for every $a \in \mathcal{A}$.

- Jensen's formula: $\Delta(a)=\Delta(\Phi(a))$ for every $a \in \mathcal{A}^{-1}$.

- Szegö's theorem: For every positive normal linear functional $\rho$ of $\mathcal{M}$,

$$
\inf \rho\left(|d+a|^{2}\right)=\inf \rho(b),
$$

where $d$ ranges over all elements of $\mathcal{D}$ with $\Delta(d) \geq 1, a$ ranges over $H_{0}^{\infty}(\mathcal{M})$, and $b$ ranges over the invertible positive operators in $\mathcal{M}$ satisfying $\exp \tau(\log b) \geq 1$.

We will prove the validity of Jensen's formula for $H^{\infty}(\mathcal{M})$, thereby establishing the validity of all three statements for $H^{\infty}(\mathcal{M})$. For a description of how the above formulation compares to the classical theorem of Szegö, the reader is referred to [A 4.4.2].

Lemma 1. Let $a, b$ be positive invertible elements of $\mathcal{M}$ with $[a, b]=0$. Then $b+b^{-1} a \geq 2 a^{1 / 2}$.

Proof. By commutativity we may multiply $0 \leq\left(b-a^{1 / 2}\right)^{2}=b^{2}+a-2 b a^{1 / 2}$ with $b^{-1}$ to get the required claim.

Lemma 2. Let $a \in \mathcal{M}$ be positive and invertible. Inductively define

$$
x_{1}=a, \quad x_{n+1}=\frac{1}{2}\left(x_{n}+a x_{n}^{-1}\right) .
$$

Then $\left(x_{n+1}\right)$ decreases monotonically to $a^{1 / 2}$ with convergence taking place in the norm topology.

Proof. Since all $x_{n}$ 's belong to the smallest $C^{*}$-algebra generated by $a$, we may pass to $C(\sigma(a))$. Then note that by the previous lemma $x_{2} \geq a^{1 / 2}$. Finally do a pointwise application of the algorithm described in [R, p 81, \#16] to see that in $C(\sigma(a)),\left(x_{n+1}\right)$ decreases monotonically to $a^{1 / 2}$. The uniform convergence follows from [R, 7.13]. 
Theorem 3 (Jensen's formula). Let $\mathcal{A}$ be a finite maximal subdiagonal subalgebra of $\mathcal{M}$. Then

$$
\Delta(a)=\Delta(\Phi(a)) \text { for each } a \in \mathcal{A}^{-1} .
$$

Proof. For any $a \in \mathcal{A}^{-1}$ the multiplicativity of the determinant ensures that $1=$ $\Delta(\mathbb{1})=\Delta(a) \Delta\left(a^{-1}\right)$. Therefore if

$$
\Delta(a) \geq \Delta(\Phi(a)) \text { for each } a \in \mathcal{A}^{-1},
$$

then since $\Phi$ is a homomorphism on $\mathcal{A}$, equality must follow since we will then also have

$$
\Delta(a)^{-1}=\Delta\left(a^{-1}\right) \geq \Delta\left(\Phi(a)^{-1}\right)=\Delta(\Phi(a))^{-1} \quad \text { for each } \quad a \in \mathcal{A}^{-1} .
$$

It therefore suffices to verify that equation (11) holds. This will in turn follow if we can show that

(2) $\tau\left(|a|^{1 / 2^{n}}\right) \geq \tau\left(|\Phi(a)|^{1 / 2^{n}}\right) \quad$ for every $a \in \mathcal{A}^{-1} \quad$ and every integer $n \geq 0$,

since the $2^{n}$-th powers of these terms will respectively decrease to $\Delta(a)$ and $\Delta(\Phi(a))$. We use induction to verify the validity of equation (2). By the $L^{1}$-contractivity of $\Phi$ (see for example [MW]) we have that

$$
\tau(|a|) \geq \tau(|\Phi(a)|) \quad \text { for every } \quad a \in \mathcal{A}^{-1} .
$$

Now suppose that for some non-negative integer $k$ we have that

$$
\tau\left(|a|^{1 / 2^{k}}\right) \geq \tau\left(|\Phi(a)|^{1 / 2^{k}}\right) \quad \text { for every } \quad a \in \mathcal{A}^{-1} .
$$

Next let $a \in \mathcal{A}^{-1}$ be given and inductively define the sequence $\left(x_{n}\right) \subset \mathcal{M}^{+}$by

$$
x_{1}=|a|^{1 / 2^{k}}, \quad x_{n+1}=\frac{1}{2}\left(x_{n}+|a|^{1 / 2^{k}} x_{n}^{-1}\right) .
$$

Then $\left(x_{n+1}\right)$ decreases monotonically in norm to $|a|^{1 / 2^{k+1}}$. Since $\mathcal{A}$ has factorisation [A. 4.2.1], we may select a sequence $\left(z_{n}\right) \subset \mathcal{A}^{-1}$ with

$$
\left|z_{n}\right|=x_{n}^{2^{k}} \quad \text { for every } \quad n \in \mathbb{N} .
$$

Now for $q=\left(\frac{1}{2}\right)^{k}$, we have by [FK, $\left.2.5 \& 2.8\right]$ that

$$
\begin{aligned}
\tau\left(\left|a z_{n}^{-1}\right|^{q}\right)^{1 / q} & =\tau\left(\left.\left.\left|z_{n}^{-1 *}\right| a\right|^{2} z_{n}^{-1}\right|^{q / 2}\right)^{1 / q} \\
& =\tau\left(\| a\left|z_{n}^{-1}\right|^{q}\right)^{1 / q} \\
& =\tau\left(\left|z_{n}^{-1 *}\right| a||^{q}\right)^{1 / q} \\
& =\tau\left(|| a\left|z_{n}^{-1} z_{n}^{-1 *}\right| a||^{q / 2}\right)^{1 / q} \\
& =\tau\left(\|\left. a|| z_{n}\right|^{-2}|a|^{q / 2}\right)^{1 / q} \\
& =\tau\left(\| a\left|x_{n}^{-2 / q}\right| a||^{q / 2}\right)^{1 / q} \\
& =\tau\left(x_{n}^{-1}|a|^{q}\right)^{1 / q} .
\end{aligned}
$$

(The last equality follows from the fact that the $x_{n}$ 's and $|a|$ belong to the same commutative $C^{*}$-subalgebra of $\mathcal{M}$.) So by the induction hypothesis we now have 
that

$$
\begin{aligned}
\tau\left(x_{n+1}\right) & =\frac{1}{2} \tau\left(x_{n}+|a|^{1 / 2^{k}} x_{n}^{-1}\right) \\
& =\frac{1}{2} \tau\left(\left|z_{n}\right|^{1 / 2^{k}}+\left|a z_{n}^{-1}\right|^{1 / 2^{k}}\right) \\
& \geq \frac{1}{2} \tau\left(\left|\Phi\left(z_{n}\right)\right|^{1 / 2^{k}}+\left|\Phi\left(a z_{n}^{-1}\right)\right|^{1 / 2^{k}}\right) \\
& =\frac{1}{2} \tau\left(\left|\Phi\left(z_{n}\right)\right|^{1 / 2^{k}}+\left|\Phi(a) \Phi\left(z_{n}\right)^{-1}\right|^{1 / 2^{k}}\right) .
\end{aligned}
$$

Now since $2^{k}+2^{k}=2^{k+1}$ we may apply Hölder's inequality [FK, 4.9] with $p=q=$ $\left(\frac{1}{2}\right)^{k}$ and $r=\left(\frac{1}{2}\right)^{k+1}$ to see that

$$
\tau\left(|\Phi(a)|^{1 / 2^{k+1}}\right)^{2^{k+1}} \leq \tau\left(\left|\Phi(a) \Phi\left(z_{n}\right)^{-1}\right|^{1 / 2^{k}}\right)^{2^{k}} \tau\left(\left|\Phi\left(z_{n}\right)\right|^{1 / 2^{k}}\right)^{2^{k}} .
$$

This in turn translates to

$$
\tau\left(\left|\Phi(a) \Phi\left(z_{n}\right)^{-1}\right|^{1 / 2^{k}}\right) \geq \frac{\tau\left(|\Phi(a)|^{1 / 2^{k+1}}\right)^{2}}{\tau\left(\left|\Phi\left(z_{n}\right)\right|^{1 / 2^{k}}\right)} .
$$

Thus by equation (4) and Lemma 1 we then have that

$$
\begin{aligned}
\tau\left(x_{n+1}\right) & \geq \frac{1}{2} \tau\left(\left|\Phi\left(z_{n}\right)\right|^{1 / 2^{k}}+\left|\Phi(a) \Phi\left(z_{n}\right)^{-1}\right|^{1 / 2^{k}}\right) \\
& \geq \frac{1}{2}\left[\tau\left(\left|\Phi\left(z_{n}\right)\right|^{1 / 2^{k}}\right)+\frac{\tau\left(|\Phi(a)|^{1 / 2^{k+1}}\right)^{2}}{\tau\left(\left|\Phi\left(z_{n}\right)\right|^{1 / 2^{k}}\right)}\right] \\
& \geq \tau\left(|\Phi(a)|^{1 / 2^{k+1}}\right) .
\end{aligned}
$$

Since $\left(x_{n+1}\right)$ decreases uniformly and monotonically to $|a|^{1 / 2^{k+1}}$, it follows that

$$
\tau\left(|a|^{1 / 2^{k+1}}\right) \geq \tau\left(|\Phi(a)|^{1 / 2^{k+1}}\right)
$$

as required. The result therefore follows.

\section{REFERENCES}

[A] W.B. Arveson, Analyticity in operator algebras, Amer. J. Math. 89(1967), 578 - 642. MR 0223899 (36:6946)

[E] R. Exel, Maximal subdiagonal algebras, Amer. J. Math. 110(1988), 775 - 782. MR0955297 (90b:46114)

[FK] T. Fack and H. Kosaki, Generalized s-numbers of $\tau$-measurable operators, Pacific J. Math. 123(1986), 269 - 300. MR0840845 (87h:46122)

[MW] M. Marsalli and G. West, Non-commutative $H^{p}$ spaces, J. Operator Theory 40(1998), 339 - 355. MR 1660390 (2000b:46117)

[R] W. Rudin, Principles of mathematical analysis: 3rd edition, McGraw-Hill, 1976. MR0385023 (52:5893)

Department of Mathematical Sciences, University of South Africa, Box 392, 0003 Unisa, South Africa

E-mail address: labusle@unisa.ac.za 